\title{
The Edinburgh/AAO/Strasbourg Catalogue of Galactic Planetary Nebulae
}

\author{
Q.A. Parker ${ }^{1,3}$, M.Hartley ${ }^{2}$, D.Russeil ${ }^{4}$, A.Acker \& F.Ochsenbein ${ }^{5}$ \\ D.H.Morgan ${ }^{1}$, S.Beaulieu ${ }^{7}$, R.Morris \& S.Phillipps ${ }^{6}$, M.Cohen ${ }^{8}$ \\ ${ }^{1}$ Institute for Astronomy, Edinburgh, UK, ${ }^{2}$ Anglo-Australian \\ Observatory, Australia \\ ${ }^{3}$ Dept of Physics, Macquarie University, NSW 2109, Australia \\ ${ }^{4}$ Marseille Observatory, France, ${ }^{5}$ Strasbourg Observatory, France \\ ${ }^{6}$ Dept Physics, Univ.Bristol, UK, ${ }^{7}$ Univ.Victoria, Victoria, Canada, ${ }^{8}$ \\ UC-Berkely, USA
}

Abstract. We report on version 1.0 of the Edinburgh/AAO/Strasbourg catalogue of new and possible Planetary Nebulae (PN) distributed via cdrom at this meeting. We provide accurate positions, designations, images and other descriptive parameters for the PN. In future releases this will be supplemented by inclusion of spectra and related material such as line ratios, velocities etc.

The $900+$ PN have been discovered solely from visual scrutiny of narrow-band exposures taken for the AAO/UKST H-alpha survey of the Southern Galactic Plane. Most have classic PN-type morphologies (i.e. bi-polar, rings, shells or ovals). SuperCOSMOS data will soon supersede our visual scanning but it proved an effective preliminary technique to identify candidate PN on the basis of morphology, isolation and identification as an $\mathrm{H}$-alpha nebulosity. We already have confirmatory spectroscopy for $\sim 700$ objects. Much of our new sample are of very low surface brightness, with no obvious central star, and so have remained undetected in previous surveys. They are revealed here due to the excellent depth, resolution, coverage and uniformity of the H-alpha survey. Many PN are also well extended. The average angular size is 51" with the median of 27 " but examples extend to several arcminutes. This may indicate many are in a highly evolved state where the central star has faded from easy optical detection and the nebula itself is dissipating into the ambient ISM. Large numbers of candidate PN have also been found in the Galactic Bulge region, most of which have been confirmed via UKST FLAIR/6dF MOS spectroscopy (Parker et al, in preparation and these proceedings).

By version 2.0 (release in 2002) we will have doubled the number of Galactic PN accrued from all sources over the last 75 years. This new catalogue should have a profound impact on many aspects of PN research. 